\title{
Relación entre el volumen plaquetario medio y la gravedad de la enfermedad arterial coronaria
}

\author{
Hamidreza Varastehravan ${ }^{1}$, Seyed Saeed Karbalaei Tafti ${ }^{2}$, Hossein Nough $^{3}$, Aryan Naghedi. ${ }^{4}$ \\ Departamento de cardiología, Hospital Shahid Sadoughi, Universidad de Ciencias Médicas Shahid Sadoughi, Yazd, Irán.
}

Fuente de financiación: La presente investigación no ha recibido financiamiento de agencias de sectores públicos o privados.

Conflicto de intereses: ninguno.

Antecedentes y objetivos: las plaquetas desempeñan un papel principal en la patogénesis de las enfermedades de las arterias coronarias. El volumen plaquetario medio (VPM) es un indicador del volumen plaquetario circulante y se ha demostrado que está relacionado con la actividad plaquetaria. El objetivo del presente estudio es investigar si el VPM está asociado con la gravedad de la enfermedad de las arterias coronarias.

Métodos: Medimos VPM en 910 pacientes consecutivos con síndrome coronario agudo sintomático sometidos a angiografía coronaria. Se recogieron los datos básicos de los pacientes. La enfermedad coronaria significativa se definió como una estenosis $>50 \%$ en al menos 1 vaso coronario y la gravedad como la enfermedad de tres vasos y/o la enfermedad del tronco de la coronaria izquierda.
Resultados: Este estudio incluyó 477 (52,4\%) hombres y $433(47,6 \%)$ mujeres. Los resultados mostraron una relación significativa entre el VPM y la gravedad de la enfermedad arterial coronaria $(\mathrm{P}=0,001)$ y un modelo de regresión logística confirmó que el aumento de cada unidad en el volumen plaquetario medio puede aumentar el riesgo de enfermedad arterial coronaria grave en $4,67 \%$. Encontramos que el VPM es mayor en los pacientes de sexo masculino y fumador de cigarrillos.

Conclusión: El VPM está relacionado con la gravedad de la enfermedad arterial coronaria, y podría ser un parámetro útil para la evaluación primaria de la gravedad del compromiso de las arterias coronarias en pacientes con síntomas de enfermedad coronaria.

Palabras clave: enfermedad arterial coronaria; volumen plaquetario medio; angiografía coronaria

Correspondencia:

Aryan Naghedi, MD.

a.naghedi@yahoo.com 


\section{Relationship between mean platelet volume and gravity of coronary artery disease}

Background and aim: Platelets play a principal role in pathogenesis of coronary artery diseases. Mean platelet volume (MPV) is an indicator of circulating platelet size and has been demonstrated to be correlated with platelet activity. The aim of the current study was to investigate whether MPV is associated with the severity of coronary artery disease.

Methods: We measured MPV in 910 consecutive patients with symptomatic acute coronary syndrome undergoing coronary angiography. The basic data of patients were collected. Significant coronary artery disease was defined as $>50 \%$ stenosis in at least 1 coronary artery and severe coronary artery disease was defined as three vessel disease and/or left main artery disease.
Results: This study included 477 (52.4\%) males and 433(47.6\%) females. A significant relationship between MPV and severity of coronary artery disease $(\mathrm{p}=0.001)$ was found. Logistic regression analysis confirmed that each unit increment in mean platelet volume was associated to a $4.67 \%$ increase in the in the risk of severe coronary artery disease. In addition, we found that MPV was higher in males and in cigarette smokers.

Conclusion: This study showed that MPV is related to severity of coronary artery disease. MPV might be a useful parameter for primary evaluation of severity of coronary artery involvement in patients with symptoms of coronary artery diseases.

Keywords: coronary artery disease; mean platelet volume; coronary angiography 


\section{Introducción:}

La enfermedad de las arterias coronarias es una de las enfermedades más comunes en todo el mundo. Se inicia siguiendo a la formación y desarrollo de placas ateroscleróticas en su interior. Esta enfermedad es una de las causas más importantes de muerte y discapacidad en los países desarrollados, llegando a un tercio del total de muertes en personas mayores de 35 años en Estados Unidos $^{1}$.

Existen diferentes factores asociados al desarrollo de la enfermedad. Uno de estos es el envejecimiento, con el cual aumentan las posibilidades de enfermedad coronaria $^{2}$. En la mayoría de los pacientes existen otros factores de riesgo ${ }^{3}$ entre los que destacan el hábito tabáquico, la obesidad abdominal, la hipertensión arterial (HTA), diabetes mellitus (DM), dislipidemia, factores psicosociales y consumo de alcohol ${ }^{4}$.

La formación de trombos seguida por la formación de placa aterosclerótica es el factor principal en la patogénesis de la enfermedad coronarias y las plaquetas desempeñan un papel importante ${ }^{5}$. Las plaquetas son células redondas y similares a discos, cuyo recuento normal oscila entre 150000 a 400000 por ml de sangre. $\mathrm{Su}$ papel es mantener la hemostasia vascular. Las plaquetas circulantes son diferentes según su tamaño y actividad hemostática y liberan muchos agentes importantes involucrados en la coagulación, la inflamación, la trombosis y la aterosclerosis ${ }^{6}$.

La edad y el tamaño de las plaquetas son factores importantes asociados a su actividad. Se ha demostrado que el tamaño y la forma de las plaquetas cambia en el momento de su formación y en el sitio de activación ${ }^{7}$.

El VPM es reconocido como un índice de la actividad plaquetaria que está involucrada en la fisiopatología de las enfermedades ateroscleróticas. Las plaquetas más grandes contienen más gránulos y receptores de adhesión que causan un menor tiempo de sangrado e indican una actividad plaquetaria aumentada ${ }^{8}$. El VPM elevado también puede ser causado por el consumo de plaquetas más pequeñas durante la isquemia aguda ${ }^{9}$.

Diversas investigaciones han demostrado una asociación estrecha entre el VPM y factores de riesgo cardiovascular como la hiperglicemia, la DM, la HTA, la hipercolesterolemia y la obesidad ${ }^{10-12}$. También se ha demostrado que la elevación del VPM se asocia a la alteración de la perfusión miocárdica ${ }^{13}$. Sin embargo, aún no se ha investigado al VPM, como un predictor de la gravedad de la enfermedad arterial coronaria.

\section{Métodos:}

En este estudio analítico descriptivo de corte transversal, incluimos a 910 pacientes sintomáticos con diagnóstico de síndrome coronario agudo referidos al laboratorio de cateterismo cardíaco del centro cardíaco Afshar, Yazd, Irán. No hubo criterios de exclusión definidos para nuestro estudio.

Todos los procedimientos fueron realizados de acuerdo con los estándares éticos del Comité de experimentación humana de la Universidad de Ciencias Médicas Shahid Sadoughi, Yazd, Irán y con la Declaración de Helsinki de 1975. Se obtuvo consentimiento informado de todos los pacientes incluidos en el estudio.

Todas las angiografías coronarias se realizaron mediante técnicas estándar a través de un catéter cobra $5 \mathrm{~F}$ y las imágenes fueron interpretadas por dos cardiólogos experimentados.

Para cada paciente, se recopilaron datos demográficos, como antecedentes de enfermedades cardiovasculares, historial de medicamentos, edad, y sexo. Luego, para cada paciente se tomó una muestra de sangre y se verificó VPM, glicemia, colesterol y triglicéridos (TG). Se extrajeron muestras de sangre de la vena antecubital después de un ayuno de 12 horas. Todas las pruebas bioquímicas de rutina se llevaron a cabo en un autoanalizador (sistemas modulares de diagnóstico de Roche, Tokio, Japón). Para el análisis de VPM, las muestras de sangre con K3 EDTA se procesaron con un analizador Sysmex XT-2000i (Sysmex, Kobe, Japón). El VPM normal se definió entre 9,4 fL y 12,3 fL. La DM se definió como glicemia en ayunas $(\mathrm{FBS}) \geq 126$ o por el antecedente de tratamiento con agentes antidiabéticos. La hiperlipidemia (HLP) o dislipidemia se definió como lipoproteína de baja densidad (LDL) $\geq 160 \mathrm{mg} / \mathrm{dl}$ o triglicéridos $(\mathrm{TG}) \geq 200 \mathrm{mg} / \mathrm{dl}$ o Colesterol total $\geq 240$ $\mathrm{mg} / \mathrm{dl}$ en una prueba de muestra de sangre, o por recibir tratamiento con agentes hipolipemiantes. Los pacientes con presión arterial $>140 / 90 \mathrm{mmHg}$ o los pacientes que recibieron agentes antihipertensivos se consideraron portadores de HTA.

Los pacientes se dividieron en tres grupos según la opinión de dos expertos cardiólogos: 1) Estenosis leve en angiografía o angiografía normal. 2) compromiso coronario significativo, que se definió como un mínimo de $50 \%$ de estenosis en al menos una de las arterias coronarias. 3) Compromiso grave, que se definió como compromiso de tres vasos o compromiso del tronco de la arteria coronaria izquierda.

Todas las variables, incluyendo edad, sexo, DM, HTN, 
dislipidemia, tabaquismo, VPM e intensidad de la enfermedad coronaria se registraron en una base de datos que se analizó con el software SPSS ${ }^{20}$. Los resultados se muestran en tablas de distribución de frecuencia.

\section{Resultados}

Entre los 910 pacientes incluidos, $477(52,4 \%)$ eran hombres y $433(47,6 \%)$ eran mujeres. El rango de edad fue de 27 a 88 años y la edad promedio fue 59,5 años.

Todos los pacientes incluidos se sometieron a angiografía y se dividieron en tres grupos según la gravedad de la enfermedad arterial coronaria. 340 pacientes $(37,4 \%)$ tenían una coronariografía normal o sin lesiones obstructivas; 269 pacientes $(29,6 \%)$ tenían una enfermedad coronaria significativa y 310 pacientes $(33,1 \%)$ tenían una enfermedad coronaria grave. Las características de los pacientes basada en la gravedad de la enfermedad arterial coronaria se resumen en la Tabla1.

\begin{tabular}{|l|c|c|}
\hline $\begin{array}{l}|l| \\
\text { Tabla 1. Distribución de pacientes basada en la severidad de } \\
\text { la enfermedad coronaria. }\end{array}$ \\
\hline
\end{tabular}

286 pacientes $(31,4 \%)$ eran diabéticos y $289(31,8 \%)$ tenían hiperlipidemia; 513 pacientes $(56,4 \%)$ tenían hipertensión.

Los pacientes fueron divididos en cuatro grupos basados en el consumo de tabaco: $600(65,9 \%)$ no consumían tabaco; 261 (28,7\%) solo consumían tabaco; 24 $(2,6 \%)$ eran adictos al opio y, finalmente, 25 pacientes $(2,7 \%)$ eran fumadores y también adictos al opio. Todos los datos de los pacientes basados en el consumo de tabaco se resumen en la Tabla 2.

En nuestro estudio investigamos la correlación entre la gravedad de la enfermedad coronaria y el VPM junto con otros factores de riesgo. Para determinar la relación con variables categóricas como género, DM, HLP, HTN y consumo de tabaco, se usó la prueba de Chi-Square y se utilizó la prueba ANOVA para comparar los promedios basados en la edad y VPM.

Hubo una correlación estadísticamente significativa en-

\begin{tabular}{l|c|c|}
$\begin{array}{l}\text { Tabla 2. Distribución de pacientes de acuerdo al consumo } \\
\text { de tabaco u adicción al opio. }\end{array}$ \\
\hline & N & $\%$ \\
\hline No consume de tabaco & 600 & $65,9 \%$ \\
\hline Fumador & 261 & $28,7 \%$ \\
\hline Adicto al opio & 24 & $2,6 \%$ \\
\hline Fumador y adicto al opio & 25 & $2,7 \%$ \\
\hline Total & 910 & $100 \%$ \\
\hline
\end{tabular}

tre la gravedad de la enfermedad coronaria y el sexo, la edad, la HLP, la HTN, el consumo de tabaco y el VPM. No se observó una correlación estadísticamente significativa entre la gravedad de la enfermedad coronaria y la DM.

El objetivo principal de nuestro estudio fue determinar la correlación entre la gravedad de la enfermedad arterial coronaria y el VPM. Usando la prueba ANO$\mathrm{VA}$, investigamos esta correlación que fue significativa $(\mathrm{p}=0.01)$. Utilizando la prueba de persecución Dunnett T3 se reveló que la diferencia entre el VPM de pacientes con coronarias normales o levemente afectadas comparados con aquello con enfermedad significativa fue significativa $(\mathrm{p}=0,001)$. La diferencia entre el promedio de VPM en pacientes con coronariografía normal o con lesiones leves (normal/leve) comparado con aquellos con enfermedad coronaria grave fue significativa $(\mathrm{p}=0,001)$. También hubo una significativa diferencia $(\mathrm{p}=0.001)$ entre el promedio de VPM en pacientes con enfermedad coronaria significativa comparados con aquellos que presentaban compromiso coronario grave.

Los detalles estadísticos sobre la correlación entre la gravedad de la enfermedad coronaria y todos los factores de riesgo previamente mencionados se resumen en la Tabla3.

Utilizamos la prueba " $t$ " para comparar las medias de VPM en función de otros factores de riesgo - género, DM, HLP y HTN -, exceptuando la gravedad de las enfermedad de las arterias coronarias. Para comparar las medias de VPM en diferentes grupos en función del consumo de tabaco, utilizamos la prueba ANOVA. Hubo una correlación estadísticamente significativa entre VPM y género o VPM y consumo de tabaco, pero no la hubo entre VPM y DM, HLP o HTN.

Para determinar factores de predicción de la gravedad de la enfermedad de las arterias coronarias se utilizó el modelo de regresión logística (Tabla 4). La enfermedad 
Tabla 3. Correlación entre el compromiso arterial coronario y los diversos factores de riesgo.

\begin{tabular}{|c|c|c|c|c|c|c|}
\hline & & $\begin{array}{c}\text { normal o } \\
\text { leve }\end{array}$ & significativo & grave & Total & $p$ \\
\hline \multirow[t]{2}{*}{ Género } & $\begin{array}{l}M \\
(\%)\end{array}$ & 44,1 & 55,0 & 59,5 & 52,4 & \multirow[t]{2}{*}{0,001} \\
\hline & $\begin{array}{c}\mathrm{F} \\
(\%)\end{array}$ & 55,9 & 45 & 40,5 & 47,6 & \\
\hline Edad & & 56,78 & 59,71 & 62,39 & 59,50 & 0,001 \\
\hline DM \% & & $31,8 \%$ & $32,7 \%$ & $29,9 \%$ & $31,4 \%$ & 0,76 \\
\hline HLP \% & & $27,9 \%$ & $27,1 \%$ & $40,2 \%$ & $31,8 \%$ & 0,001 \\
\hline HTN \% & & $35,9 \%$ & $41,6 \%$ & $54,2 \%$ & $43,6 \%$ & 0,001 \\
\hline $\begin{array}{l}\text { Sin tabaco } \\
\text { ni opio }\end{array}$ & & $72,9 \%$ & $: 69,1 \%$ & $55,1 \%$ & $65,9 \%$ & \multirow{4}{*}{0,001} \\
\hline Fumador & & $24,1 \%$ & $27,1 \%$ & $35,2 \%$ & $28,7 \%$ & \\
\hline Opio & & $1,5 \%$ & $2,2 \%$ & $4,3 \%$ & $2,6 \%$ & \\
\hline $\begin{array}{l}\text { Fumador y } \\
\text { opio }\end{array}$ & & $1,5 \%$ & $1,5 \%$ & $5,3 \%$ & $2,7 \%$ & \\
\hline $\begin{array}{l}\text { Nivel medio } \\
\text { de VPM (fL) }\end{array}$ & & 9,18 & 10,13 & 11,15 & 10,11 & 0,001 \\
\hline
\end{tabular}

M: masculino; F: femenino; DM: diabetes mellitus; HLP: hiperlipidemia; HTN: hipertensión arterial.

Tabla 4. Análisis de regresión logística: variables predictoras de severidad del compromiso coronario.

\begin{tabular}{|l|c|c|c|c|}
\hline Variable & B & S.E. & $p$ & Exp (B) \\
\hline Edad & 0,01 & 0,19 & 0,035 & 1,01 \\
\hline Género & $-0,19$ & 0,19 & 0,33 & 0,82 \\
\hline VPM & 1,54 & 0,108 & 0,001 & 4,67 \\
\hline HLP & 0,09 & 0,2 & 0,64 & 1,1 \\
\hline HTN & 0,64 & 0,19 & 0,001 & 1,91 \\
\hline Fumar & 0,14 & 0,22 & 0,53 & 1,15 \\
\hline Adicción & 0,41 & 0,63 & 0,51 & 1,51 \\
\hline Fumar + Adicción & 0,49 & 0,67 & 0,46 & 1,64 \\
\hline
\end{tabular}

de la arteria coronaria se consideró una variable dependiente y la población de estudio se dividió en 2 grupos: 1) aquellos en que la enfermedad coronaria era leve o ausente, y 2) aquellos que tenían enfermedad coronaria significativa o grave. Las variables género, edad, VPM, HLP, HTN y consumo de tabaco se introdujeron en el modelo de regresión logística. Los resultados revelaron que el VPM, la HTN y la edad fueron factores para predecir la gravedad de la enfermedad arterial coronaria $(\mathrm{p}=0,001)$.

Además, pudo estimarse que, por cada incremento de un año en la edad, la probabilidad de enfermedad coronaria grave aumenta en $1,01 \%(\mathrm{p}=0,003)$.

La presencia de hipertensión confiere un riesgo 1,9 veces mayor de presentar enfermedad coronaria sig- 
nificativa o grave con respecto a la población normal $(p=0,001)$. Finalmente, por cada incremento de un femtolitro en VPM, la probabilidad de enfermedad coronaria significativa o grave aumenta en $4,67 \%(\mathrm{p}=0,001)$.

\section{Discusión:}

Este estudio mostró que la gravedad de la enfermedad arterial coronaria se correlaciona con el VPM y cada incremento unitario de VPM (en femtolitros) aumenta el riesgo de enfermedad arterial coronaria grave en 4,67\%. También se demostró que la gravedad de la enfermedad arterial coronaria se correlaciona con la edad, el sexo, la HLP, la HTN y el consumo de tabaco, pero no se correlaciona con la DM. Nuestro estudio mostró que el VPM está correlacionado con el género y el consumo de tabaco, pero no está correlacionado con las otras variables estudiadas.

En un estudio, Slavka et al demostraron que el VPM se correlaciona con la gravedad de la enfermedad de las arterias coronaria, lo que concuerda con lo encontrado en esta investigación ${ }^{14}$.

También otros estudios realizados por Khode et al y Endler et al en 2012 y 2002 respectivamente, concuerdan con los resultados del presente estudio ${ }^{15,16}$. La correlación de mayor VPM con gravedad de la enfermedad coronaria puede deberse al consumo de plaquetas más pequeñas durante la formación de la placa aterosclerótica y al reemplazo de plaquetas pequeñas con plaquetas más grandes que tienen un nivel de actividad más alto.

A diferencia de los resultados de los trabajos mencionados anteriormente y los de la presente investigación, De Luca et al en 2009 no encontraron correlación entre el VPM y la gravedad de la enfermedad de las arterias coronarias ${ }^{17}$.

En el presente estudio se encontró que el consumo de tabaco se correlaciona significativamente con el VPM, coincidiendo con lo comunicado por Kario et al en $1992^{18}$. Esta asociación puede deberse a un proceso acelerado de aterosclerosis asociado al tabaquismo, que produciría un mayor consumo de plaquetas más pequeñas, lo que conduciría a su reemplazo por megacariocitos.

Nuestro estudio mostró que no existe una relación significativa entre la HTN y el VPM. En estudios realizados por Pathansali et al y Coban et al en 2001 y 2005, respectivamente, se llegó a la conclusión de que en pacientes con HTN, el VPM fue significativamente mayor que en los pacientes sin HTN, resultado opuesto a lo aquí presentado ${ }^{19}, 20$. Esta discrepancia no puede ex- plicarse por el bajo número de sujetos incluidos en esas investigaciones (20 y 36 respectivamente), comparado con el número de 397 pacientes hipertensos incluidos en este estudio.

Nuestro estudio mostró una relación significativa entre la gravedad de la enfermedad coronaria y la edad, el sexo, la HLP, la HTN y el consumo de tabaco. En otros estudios, como el estudio de Hennekens et al en 1998 y los estudios de Khot, Greenland y Kanto que se realizaron por separado en 2003, se informó que la relación entre la gravedad del compromiso de las arterias coronarias y las mismas variables exploradas en el presente estudio fue similar ${ }^{2}, 3,21$. El envejecimiento aumenta el riesgo de enfermedad grave de las arterias coronarias y esto puede deberse a un proceso aterosclerótico más prolongado y crónico en pacientes mayores. El género masculino aumenta el riesgo del compromiso de las arterias coronarias lo que puede deberse a factores ambientales, regímenes alimentarios, mayor consumo de cigarrillos y una mayor tendencia genética a la aterosclerosis en comparación con las mujeres ${ }^{22}$.

En muchos estudios se ha concluido que un mayor nivel de lipoproteínas de alta densidad tiene un efecto protector en el proceso de aterosclerosis, en tanto que un mayor nivel de lipoproteínas de baja densidad, TG y colesterol acelera el proceso de aterosclerosis. En base a esto, la hiperlipidemia (HLP) puede elevar el riesgo de enfermedades cardiovasculares debido a una alteración del perfil lipídico ${ }^{23}$.

La HTN también puede aumentar el riesgo de enfermedad de las arterias coronarias por efecto crónico en las paredes de los vasos y en las placas ateroscleróticas ${ }^{24}$. El consumo de tabaco también puede aumentar el riesgo de enfermedad de las arterias coronarias lo que puede deberse a que se afecta el perfil lipídico, los factores de coagulación y la actividad plaquetaria ${ }^{25}$.

\section{Conclusión:}

El presente estudio muestra una correlación estadísticamente significativa entre el VPM y la gravedad de la enfermedad arterial coronaria. Creemos que el VPM puede usarse para estimar el riesgo de encontrar una enfermedad coronaria significativa o grave en pacientes sintomáticos con síndrome coronario agudo.

Nuestro estudio necesita ser confirmado por otros y, especialmente, por ensayos clínicos que investiguen si el uso de más agentes antiplaquetarios disminuye el riesgo de presentar síndrome coronario agudo o su gravedad. 


\section{Referencias}

1. MEMBERS WG, LLOYD-JONES D, ADAMS RJ, BROWN TM, CARNETHON M, DAI S, et al. Executive summary: heart disease and stroke statistics -2010 update: a report from the American Heart Association. Circulation. 2010;121(7):948-54.

2. KHOT UN, KHOT MB, BAJZER CT, SAPP SK, OHMAN EM, BRENER SJ, et al. Prevalence of conventional risk factors in patients with coronary heart disease. Jama. 2003;290(7):898904.

3. GREENLAND P, KNOLL MD, STAMLER J, NEATON JD, DYER AR, GARSIDE DB, et al. Major risk factors as antecedents of fatal and nonfatal coronary heart disease events. Jama. 2003;290(7):891-7.

4. YUSUF S, HAWKEN S, ÔUNPUU S, DANS T, AVEZUM A, LANAS F, et al. Effect of potentially modifiable risk factors associated with myocardial infarction in 52 countries (the INTERHEART study): case-control study. The lancet. 2004;364(9438):937-52.

5. VARASTEHRAVAN H, NAGHEDI A, NOUGH H, POURMIRAFZALI H. The relationship between severity of coronary artery disease and mean platelet volume2019.

6. GAWAZ M, LANGER H, MAY AE. Platelets in inflammation and atherogenesis. The Journal of clinical investigation. 2005;115(12):3378-84.

7. A. JAGROOP IC, J. LEWIN, DP MIKHAILIDIS, I. Shape change in human platelets: measurement with a channelyzer and visualisation by electron microscopy. Platelets. 2000;11(1):2832.

8. TSIARA S, ELISAF M, JAGROOP IA, MIKHAILIDIS DP. Platelets as predictors of vascular risk: is there a practical index of platelet activity? Clinical and Applied Thrombosis/Hemostasis. 2003;9(3):177-90.

9. PIZZULLI L, YANG A, MARTIN J, LÜDERITZ B. Changes in platelet size and count in unstable angina compared to stable angina or non-cardiac chest pain. European heart journal. 1998;19(1):80-4.

10. COBAN E, OZDOGAN M, YAZICIOGLU G, AKCIT F. The mean platelet volume in patients with obesity. International journal of clinical practice. 2005;59(8):981-2.

11. NADAR SK, BLANN AD, KAMATH S, BEEVERS DG, LIP GY. Platelet indexes in relation to target organ damage in hi- gh-risk hypertensive patients: a substudy of the Anglo-Scandinavian Cardiac Outcomes Trial (ASCOT). Journal of the American College of Cardiology. 2004;44(2):415-22.

12. HEKIMSOY Z, PAYZIN B, ÖRNEK T, KANDOĞAN G. Mean platelet volume in Type 2 diabetic patients. Journal of Diabetes and its Complications. 2004;18(3):173-6.

13. SEZER M, OKCULAR I, GOREN T, OFLAZ H, NISANCI Y, UMMAN B, et al. Association of haematological indices with the degree of microvascular injury in patients with acute anterior wall myocardial infarction treated with primary percutaneous coronary intervention. Heart. 2007;93(3):313-8.

14. SLAVKA G, PERKMANN T, HASLACHER H, GREISENEGGER S, MARSIK C, WAGNER OF, et al. Mean platelet volume may represent a predictive parameter for overall vascular mortality and ischemic heart disease. Arteriosclerosis, thrombosis, and vascular biology. 2011;31(5):1215-8.

15. KHODE V, SINDHUR J, KANBUR D, RUIKAR K, NALLULWAR S. MEAN platelet volume and other platelet volume indices in patients with stable coronary artery disease and acute myocardial infarction: A case control study. Journal of cardiovascular disease research. 2012;3(4):272-5.

16. ENDLER G, KLIMESCH A, SUNDERDPLASSMANN H, SCHILLINGER M, EXNER M, MANNHALTER C, et al. Mean platelet volume is an independent risk factor for myocardial infarction but not for coronary artery disease. British journal of haematology. 2002;117(2):399-404.

17. DE LUCA G, SANTAGOSTINO M, SECCO GG, CASSETTI E, GIULIANI L, FRANCHI E, et al. Mean platelet volume and the extent of coronary artery disease: results from a large prospective study. Atherosclerosis. 2009;206(1):292-7.

18. KARIO K, MATSUO T, NAKAO K. Cigarette smoking increases the mean platelet volume in elderly patients with risk factors for atherosclerosis. Clinical \& Laboratory Haematology. 1992;14(4):281-7.

19. PATHANSALI R, SMITH NM, BATH PM. Prothrombotic megakaryocyte and platelet changes in hypertension are reversed following treatment: a pilot study. Platelets. 2001;12(3):144-9.

20. COBAN E, YAZICIOGLU G, BERKANT AVCI A, AKCIT F. The mean platelet volume in patients with essential and white coat hypertension. Platelets. 2005;16(7):435-8. 
21. HENNEKENS CH. Increasing burden of cardiovascular disease: current knowledge and future directions for research on risk factors. Circulation. 1998;97(11):1095-102.

22. NAJJAR SS, SCUTERI A, LAKATTA EG. Arterial aging: is it an immutable cardiovascular risk factor? Hypertension. 2005;46(3):454-62.

23. DAHLEN GH, GUYTON JR, ATTAR M, FARMER JA, KAUTZ JA, GOTTO JR A. Association of levels of lipoprotein Lp (a), plasma lipids, and other lipoproteins with coro- nary artery disease documented by angiography. Circulation. 1986;74(4):758-65

24. VASAN RS, LARSON MG, LEIP EP, EVANS JC, O'DONNELL CJ, KANNEL WB, et al. Impact of high-normal blood pressure on the risk of cardiovascular disease. New England journal of medicine. 2001;345(18):1291-7.

25. AMBROSE JA, BARUA RS. The pathophysiology of cigarette smoking and cardiovascular disease: an update. Journal of the American college of cardiology. 2004;43(10):1731-7. 